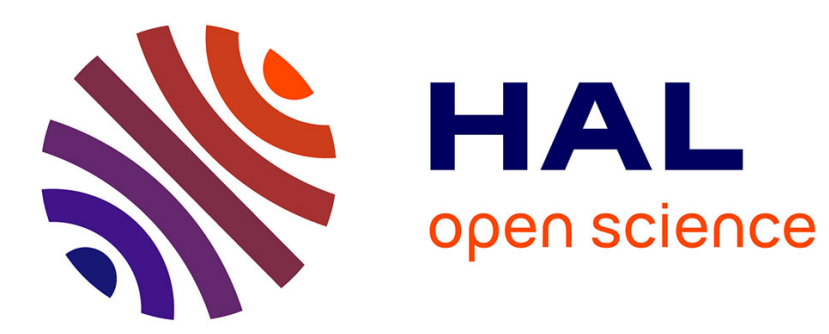

\title{
Low-temperature photoluminescence properties of Nd-doped silicon oxide thin films containing silicon nanocrystals
}

\author{
Emilie Steveler, H. Rinnert, M. Vergnat
}

\section{- To cite this version:}

Emilie Steveler, H. Rinnert, M. Vergnat. Low-temperature photoluminescence properties of Nd-doped silicon oxide thin films containing silicon nanocrystals. Journal of Luminescence, 2016, 183, pp.311314. 10.1016/j.jlumin.2016.11.048 . hal-02319426

\author{
HAL Id: hal-02319426 \\ https://hal.science/hal-02319426
}

Submitted on 17 Oct 2019

HAL is a multi-disciplinary open access archive for the deposit and dissemination of scientific research documents, whether they are published or not. The documents may come from teaching and research institutions in France or abroad, or from public or private research centers.
L'archive ouverte pluridisciplinaire HAL, est destinée au dépôt et à la diffusion de documents scientifiques de niveau recherche, publiés ou non, émanant des établissements d'enseignement et de recherche français ou étrangers, des laboratoires publics ou privés. 


\title{
Low-temperature photoluminescence properties of Nd-doped silicon oxide thin films containing silicon nanocrystals
}

\author{
E. Steveler ${ }^{1, *}$, H. Rinnert ${ }^{2}$ and M. Vergnat ${ }^{2}$ \\ ${ }^{1}$ ICube Laboratory, INSA de Strasbourg, 23 rue du Lœss BP 20 CR, 67037 Strasbourg Cedex 2, \\ France \\ ${ }^{2}$ Institut Jean Lamour, Université de Lorraine, CNRS UMR 7198, Boulevard des Aiguillettes, \\ Vandœuvre-lès-Nancy F-54500, France
}

\begin{abstract}
The luminescence properties of neodymium-doped silicon oxide thin films containing silicon nanocrystals ( $\mathrm{Si}$-nc) were studied as a function of temperature from 10 to $300 \mathrm{~K}$ by steady-state and time-resolved photoluminescence (PL) spectrometry. The Nd-related emission at $920 \mathrm{~nm}$, induced by the ${ }^{4} \mathrm{~F}_{3 / 2} \rightarrow{ }^{4} \mathrm{I}_{9 / 2}$ shell transitions, was obtained either with a resonant excitation at $585 \mathrm{~nm}$ or with an indirect excitation at $325 \mathrm{~nm}$ via Si-nc, which act as sensitizers. A saturation of the neodymiumrelated photoluminescence intensity has been evidenced for indirect excitation thanks to silicon nanocrystals at temperatures below $100 \mathrm{~K}$. According to the Förster model of energy transfer, this saturation is explained by a decrease of the coupling efficiency between Si-nc and rare earth ions at low temperatures, induced by the increase of the silicon nanocrystals lifetime at low temperatures.
\end{abstract}

Keywords : Photoluminescence, neodymium, rare earth, silicon oxide, silicon nanocrystals, lowtemperature

1. Introduction

\footnotetext{
* Corresponding author : e-mail address emilie.steveler@insa-strasbourg.fr Tel. : +33388106330

Fax: +33 388106548
} 
Silicon-based materials doped with rare earths (RE) ions have attracted much attention because of their potential use for optoelectronics [1]. Indeed the use of RE ions can give rise to light emissions at wavelengths from the far-infrared to the ultraviolet spectral range. The light emission from the RE generally arises from intrashell transitions of $4 f$ electrons which are, in first order, electric dipole forbidden. Hence, a resonant excitation of the RE leads to a weak luminescence efficiency. However, an indirect excitation mechanism can lead to a strong increase of the excitation efficiency of the optically active ions. For instance, $\mathrm{Er}^{3+}$ ions which are used in optical communications for their luminescence at $1.54 \mu \mathrm{m}$, can be indirectly excited in silica containing silicon nanocrystals ( $\mathrm{Si}-\mathrm{nc})$, thanks to an efficient energy transfer process from $\mathrm{Si}$-nc to $\mathrm{Er}^{3+}$ ions $[2$, 3, 4, 5]. The Er-related photoluminescence (PL) is thereby strongly improved since the absorption cross section of the Si-nc, which act as sensitizers, is several orders of magnitude higher than that of direct Er excitation. Similarly to the case of erbium, $\mathrm{Nd}^{3+}$ ions present several technologically important luminescent bands including the ${ }^{4} \mathrm{~F}_{3 / 2} \rightarrow{ }^{4} \mathrm{I}_{11 / 2}, \quad{ }^{4} \mathrm{~F}_{3 / 2} \rightarrow{ }^{4} \mathrm{I}_{9 / 2}$ and ${ }^{4} \mathrm{~F}_{3 / 2} \rightarrow{ }^{4} \mathrm{I}_{13 / 2} \quad 4 f$-shell transitions which are, respectively, the basis of $1.05 \mu \mathrm{m}$ Nd lasers, a second lasing transition at 900 $\mathrm{nm}$, and a 1.3-1.4 $\mu \mathrm{m}$ band in the second fiber transparency window. Because of the emission of $\mathrm{Nd}^{3+}$ ions in the near infrared range, Nd-doped materials are also of potential interest for the achievement of down-shifting or down-conversion layers, which are expected to improve the of solar cells efficiency by reducing the thermalization process of photogenerated carriers. Nd-related PL bands have been observed in silicon oxide alloys prepared by plasma-enhanced chemical vapor deposition [6], sputtering [7, 8] or evaporation [9]. In a previous work [10], we demonstrated that silicon nanocrystals are efficient sensitizers of $\mathrm{Nd}^{3+}$ ions at room temperature, but no result has been reported on the energy transfer behavior at low temperature. In the present paper, we address in the low-temperature PL properties of Nd-doped silicon oxide thin films containing Si-nc. In samples containing Si-nc as porous silicon [11] or Si-nc obtained by ionic implantation of silicon in silica [12], it has been shown that the temperature dependance of Si-nc PL intensity has a maximum around $100 \mathrm{~K}$. For Si-nc obtained by laser-induced decomposition of silane [13] or by plasma- 
enhanced chemical vapor deposition [14], the maximum of the PL of Si-nc is observed around 150 $\mathrm{K}$. The decrease of the PL intensity above $100-150 \mathrm{~K}$ is generally attributed to thermally activated non-radiative desexcitation processes. However, the generally observed decrease of the PL of Si-nc below $100 \mathrm{~K}$ is explained by a saturation effect which is due to the strong increase of the PL radiative lifetime at low temperature $[15,16,17]$.

In this work, we studied the low-temperature PL properties of Nd-doped silicon rich silicon oxide thin films prepared by evaporation. It is demonstrated that the Nd-related PL saturates at low temperatures in presence of $\mathrm{Si}-\mathrm{nc}$, only as $\mathrm{Nd}^{3+}$ ions are indirectly excited. The results are discussed in relation with the desexcitation and the excitation processes of the rare earth ions, in relation with the temperature dependence of the Si-nc PL properties.

\section{Experimental details}

The films were prepared by co-evaporation of $\mathrm{SiO}$ powder from a thermal cell and of $\mathrm{SiO}_{2}$ powder from an electron beam gun in a high-vacuum chamber with a base pressure equal to $10^{-8}$ Torr. The deposition rate equal to $0.1 \mathrm{~nm} / \mathrm{s}$ was controlled by a quartz microbalance system and the prepared samples had the composition $\mathrm{SiO}_{1.5}$. The thickness of the films was equal to $200 \mathrm{~nm}$. The $\mathrm{Nd}$ evaporation was performed from an effusion cell, heated around $1200{ }^{\circ} \mathrm{C}$. The $\mathrm{Nd}$ concentration, defined by $\mathrm{C}_{\mathrm{Nd}}=[\mathrm{Nd}] /\left[\mathrm{SiO}_{\mathrm{x}}\right]$, was calibrated by adjusting the relative deposition rates of $\mathrm{SiO}$ powder and $\mathrm{Nd}$, and was varied from 0.15 to 2 atomic $\%$. For $\mathrm{Nd}$ concentrations higher than 0.5 at.\% a PL quenching effect due to rare earth clustering is observed; however, no matter the $\mathrm{Nd}$ concentration, our photoluminescence experiments evidence the low temperature saturation of the Nd-related PL in presence of Si-nc. Thus, we only present here the results for a neodymium concentration equal to 1 atomic $\%$. The silicon substrates were maintained at $100{ }^{\circ} \mathrm{C}$ during the deposition. The Si clusters are generated by annealing post-treatments, which involves in the demixtion of the $\mathrm{SiO}_{\mathrm{x}}$ film following the reaction $\mathrm{SiO}_{\mathrm{x}} \rightarrow \mathrm{Si}+\mathrm{SiO}_{2}$. Further information on the $\mathrm{SiO}_{\mathrm{x}}$ demixtion and the formation of Si-ncs such as Fourier Transform InfraRed spectroscopy and 
Transmission Electron Microscopy (images and diffraction) can be found in previous publications $[18,19,20]$. For this study, the samples were annealed at $1100{ }^{\circ} \mathrm{C}$ during 5 minutes under a nitrogen flow in a rapid thermal furnace.

For PL intensity measurements, the samples were excited by a $30 \mathrm{~mW} \mathrm{He}-\mathrm{Cd}$ laser using the $325 \mathrm{~nm}$ line or by an optical parametric oscillator (OPO) laser at $420 \mathrm{~nm}$ or $585 \mathrm{~nm}$. The OPO laser pulse frequency and the duration were typically equal to $10 \mathrm{~Hz}$ and $5 \mathrm{~ns}$, respectively. For experiments using the OPO laser, the PL emission from the samples was obtained by integrating the time-dependent luminescence. For the time-resolved PL experiments, the samples were pumped by the $355 \mathrm{~nm}$ line of a frequency-tripled YAG:Nd laser. The laser pulse frequency, energy, and duration were typically equal to $10 \mathrm{~Hz}, 50 \mu \mathrm{J}$, and $20 \mathrm{~ns}$, respectively. The time response of the detection system was better than $1 \mu \mathrm{s}$. The PL signal was analyzed by a monochromator equipped with a 600 grooves/mm grating and by an InP/InGaAs photomultiplier tube cooled at $190 \mathrm{~K}$, with a detection range in the 600-1700 $\mathrm{nm}$ range. The response of the detection systems was precisely calibrated with a tungsten wire calibration source. PL experiments were performed at temperatures between $10 \mathrm{~K}$ and $300 \mathrm{~K}$ in a cryostat cooled with liquid helium.

\section{Results and discussion}

The PL spectra of Nd-doped silicon oxide thin films containing Si-nc are presented in Fig. 1 as a function of the temperature between 10 and $300 \mathrm{~K}$. The excitation line was non resonant with electronic levels of neodymium ions. These spectra show a broad band around $700-750 \mathrm{~nm}$, corresponding to the PL of Si-nc due to the quantum confinement, and the Nd-related PL band at $920 \mathrm{~nm}$, corresponding to the ${ }^{4} \mathrm{~F}_{3 / 2} \rightarrow{ }^{4} \mathrm{I}_{9 / 2}$ transition. The PL intensity of Si-nc shows a maximum between 90 and $150 \mathrm{~K}$, as previously seen in the literature [11-14], and the Nd-related PL intensity reaches a maximum value for temperatures below $150 \mathrm{~K}$. In order to better analyze these results, we plotted the integrated Nd-related PL intensity as a function of the temperature (Fig. 2 (a)). On the one hand, this graph shows a linear increase of the intensity of $\mathrm{Nd}^{3+}$ ions as the temperature decreases from 300 to $100 \mathrm{~K}$, due to a decrease of the non radiative recombination rate with 
lowering temperature. On the other hand, below $100 \mathrm{~K}$, the Nd-related PL intensity saturates.

To explain this low-temperature saturation of rare earth ions, we first studied the desexcitation mechanism of the neodymium ions by extracting the decay time of the rare earth. Fig. 2 (a) also presents the decay time of $\mathrm{Nd}^{3+}$ ions as a function of the temperature. Neodymium decay times were calculated by fitting the experimental PL decay curve at $920 \mathrm{~nm}$ with a single exponential decay curve. The decay time of $\mathrm{Nd}^{3+}$ ions increases almost linearly from 125 to $310 \mu \mathrm{s}$ as the temperature drops from 300 to $10 \mathrm{~K}$. This decay time can be described by the following relation:

$$
\frac{1}{\tau_{N d}}=\frac{1}{\tau_{\text {rad }}}+\frac{1}{\tau_{\text {nonrad }}}
$$

where $\tau_{N d}$ is the measured decay time of $\mathrm{Nd}^{3+}$ ions, $\tau_{\text {rad }}$ is the radiative decay time and $\tau_{\text {nonrad }}$ is the non-radiative decay time. It can be assumed that the radiative decay time does not depend on the temperature, then the increase of the measured $\mathrm{Nd}$ decay time at low temperature is due to an increase of the non-radiative decay time. Since non-radiative processes are expected to be thermally activated, non-radiative recombination rate decreases at low temperature. This indeed leads to an increase of the non-radiative decay time at low temperature and thus an increase of measured $\mathrm{Nd}$ decay time. This should involve an increase of the Nd-related PL intensity, as observed by Seo et al. [6]. In the case of our samples, the observed saturation of the Nd-related PL intensity below $100 \mathrm{~K}$ can not therefore be explained by the desexcitation mechanism of the rare earth.

Since we previously show that the desexcitation mechanism of $\mathrm{Nd}$ can not be at the origin of the Nd-related PL saturation at low temperature, the excitation mechanism of $\mathrm{Nd}^{3+}$ ions could then be the cause of this saturation. In such samples containing Si-nc, we previously demonstrated that $\mathrm{Nd}^{3+}$ ions are indirectly excited by Si-nc thanks to an efficient energy transfer [10]. We studied then the correlation between the intensities of $\mathrm{Si}-\mathrm{nc}$ and $\mathrm{Nd}^{3+}$ ions as a function of the temperature. Figure 2 (b) presents the PL intensity of Si-nc as a function of the temperature and shows that the maximum of PL is obtained around $120 \mathrm{~K}$. We can notice that the decrease of the PL intensity of 
Si-nc at low temperature and the Nd-related PL saturation occur at temperatures very close, suggesting that these phenomena are correlated. Thus, in order to understand the evolution of the Nd-related PL intensity as a function of temperature, we studied the temperature dependence of the PL of Si-nc.

Then, from the time resolved PL spectra of Si-nc for different temperatures, we extracted the decay time of Si-nc (Fig. 2 (b)). The decay time of Si-nc was extracted using a stretchedexponential law $\left(I_{P L}=I_{0} \exp (-t / \tau)^{\beta}\right)$, where $\beta$ was equal to 0.8 . Such a law is generally used to describe the time dependance of the Si-nc PL [21, 22]. On the one hand, as the temperature drops from 300 to $90 \mathrm{~K}$, the decay time of Si-nc increases slowly from 90 to $260 \mu$ s because of the decrease of the non-radiative recombination rate, explaining the increase of the PL intensity. On the other hand, the decay time of Si-nc strongly increases below $90 \mathrm{~K}$, reaching a value of $2 \mathrm{~ms}$ at $10 \mathrm{~K}$. This sharp rise is due to an important increase of the radiative decay time of $\mathrm{Si}-\mathrm{nc}$, as predicted by Calcott et al. [23]. This strong increase of the radiative decay time leads to a significant reduction of the radiative recombination efficiency, explaining the decrease of the PL intensity of Si-nc below $90 \mathrm{~K}$.

According to the Förster model on the energy transfer from a donor to an acceptor [24], a decrease of the radiative recombination rate of the donor leads to a decrease of the energy transfer probability. Since the radiative recombination efficiency of Si-nc strongly decreases at low temperatures, the probability of energy transfer from $\mathrm{Si}$-nc to $\mathrm{Nd}^{3+}$ ions also drops and leads to a decrease of the coupling efficiency at low temperature. The competition between the decrease of the coupling efficiency and the decrease of non-radiative recombination explains the saturation of the Nd-related PL.

To confirm the previous results showing the role of indirect excitation via Si-nc in the saturation of Nd-related PL at low temperature, we compared the evolution of the normalized PL intensities of the rare earth at $920 \mathrm{~nm}$ for direct and indirect excitations as a function of the temperature (Fig. 3). For direct excitation, the excitation line $\left(585 \mathrm{~nm}\right.$ ) was resonant with the ${ }^{4} \mathrm{G}_{5 / 2}$ 
and ${ }^{2} \mathrm{G}_{7 / 2}$ electronic levels of neodymium ions and, for indirect excitation, the excitation line was non resonant with electronic levels of neodymium ions. For both direct and indirect excitations, PL intensities have been normalized in order to compare the evolution of the intensity as a function of the temperature. Actually, the Nd-related PL intensity obtained for non resonant excitation through Si-nc at $325 \mathrm{~nm}$ is much higher than the one obtained for resonant excitation of the rare earth at 585 nm. This can be explained by the absorption cross section of Si-nc which is several orders of magnitude higher than the one of $\mathrm{Nd}$. [10] As previously seen in Fig. 2 (a), the Nd-related PL saturates below $100 \mathrm{~K}$ for an indirect excitation thanks to Si-nc; on the contrary, for resonant excitation, the Nd-related PL linearly increases as the temperature drops from 300 to $10 \mathrm{~K}$ and does not saturate. This result confirms that the origin of the saturation of Nd-related PL at low temperatures is the indirect excitation of the rare earth via Si-nc.

Indirect excitation thanks to amorphous Si nanoparticles could also lead to a saturation of the Nd-related PL intensity at low temperature. Thus, we studied a Nd-doped silicon oxide thin film annealed at $800{ }^{\circ} \mathrm{C}$ where Si nanoparticles were generated by demixtion. The room-temperature PL spectrum of this sample (inset of Fig. 4) shows the Nd-related PL band around $920 \mathrm{~nm}$ but the PL of Si-nc generally observed around $700-800 \mathrm{~nm}$ is not seen, revealing that the Si nanoparticles are amorphous at $800{ }^{\circ} \mathrm{C}$. In this sample without $\mathrm{Si}-\mathrm{nc}$, we demonstrated in a previous paper [20] that the indirect excitation is due to the existence of electronic defect states or amorphous Si clusters. Fig. 4 presents the Nd-related PL intensity as a function of the temperature for an excitation line which is non-resonant with electronic levels of neodymium. For this sample containing no Si-nc, the indirect excitation of $\mathrm{Nd}^{3+}$ ions leads to a linear rise of the Nd-related PL intensity as the temperature decreases from 300 to $10 \mathrm{~K}$. Unlike the indirect excitation thanks to Si-nc, indirect excitation of neodymium via electronic defect states or amorphous $\mathrm{Si}$ clusters do not lead to saturation of Nd-related PL intensity at low temperatures. This result shows that the indirect excitation of $\mathrm{Nd}^{3+}$ ions leads to the saturation of their PL only when it occurs thanks to Si-nc, because of the strong decrease of their radiative recombination efficiency at low temperatures. 
Finally, according to the Förster model, the radiative recombination rate of the Si-nc has to be high to obtain an efficient coupling and an intense Nd-related PL. Nevertheless, if the radiative recombination rate of the donor is very high, we can wonder if Si-nc do not emit luminescence preferentially rather than transferring their energy to $\mathrm{Nd}^{3+}$ ions. Thus, to obtain a strong Nd-related PL, the optimal configuration is certainly a compromise between the radiative processes of Si-nc and the coupling with the rare earth that compete with each other.

\section{Conclusions}

We studied the low-temperature PL properties of Nd-doped silicon oxide thin films containing $\mathrm{Si}$-nc elaborated by co-evaporation of $\mathrm{SiO}$ and $\mathrm{SiO}_{2}$ powders. We showed that the $\mathrm{Nd}-$ related PL intensity is strongly dependent on the temperature and that the temperature dependence of the PL intensity is different depending on the excitation process (direct or indirect). Above 100 $\mathrm{K}$, the PL of the rare earth strongly depends on thermally activated non-radiative recombinations; then, the Nd-related PL intensity increases as the temperature drops from 300 to $100 \mathrm{~K}$. On the contrary, below $100 \mathrm{~K}$, the PL intensity of neodymium saturates and is almost constant. We explained this result by the indirect excitation of the $\mathrm{Nd}^{3+}$ ions via silicon nanocrystals whose PL intensity also saturates below $100 \mathrm{~K}$. Indeed, the strong increase of the radiative decay time of Si-nc at low temperatures leads to a significant reduction of the radiative recombination rate of the nanoclusters, and, according to the Förster model, results in a decrease of the energy transfer from Si-nc to $\mathrm{Nd}^{3+}$ ions. Silicon nanocrystals, which act as sensitizers for $\mathrm{Nd}^{3+}$ ions, are then less efficient at temperatures below $100 \mathrm{~K}$. On the contrary, in absence of Si-nc (sample annealed at 800 ${ }^{\circ} \mathrm{C}$ ), when the indirect excitation occurs thanks to electronic defect states or amorphous Si clusters, the Nd-related PL intensity does not saturate and increases as the temperature drops to $10 \mathrm{~K}$. In this case, the sensitizers do not lose their efficiency at low temperatures. 


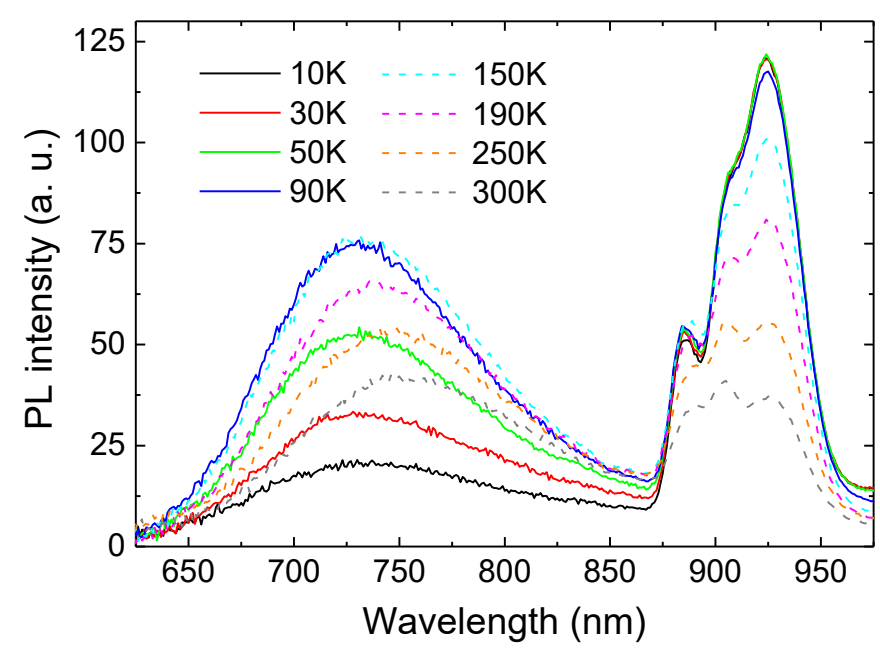

Figure 1: (Color online) CW-PL spectra of Nd-doped silicon oxide thin films containing $\mathrm{Si}$ nanocrystals as a function of the wavelength for different temperature measurements ranging from $10 \mathrm{~K}$ to $300 \mathrm{~K}$. The spectra are obtained with an indirect excitation at $325 \mathrm{~nm}$. 


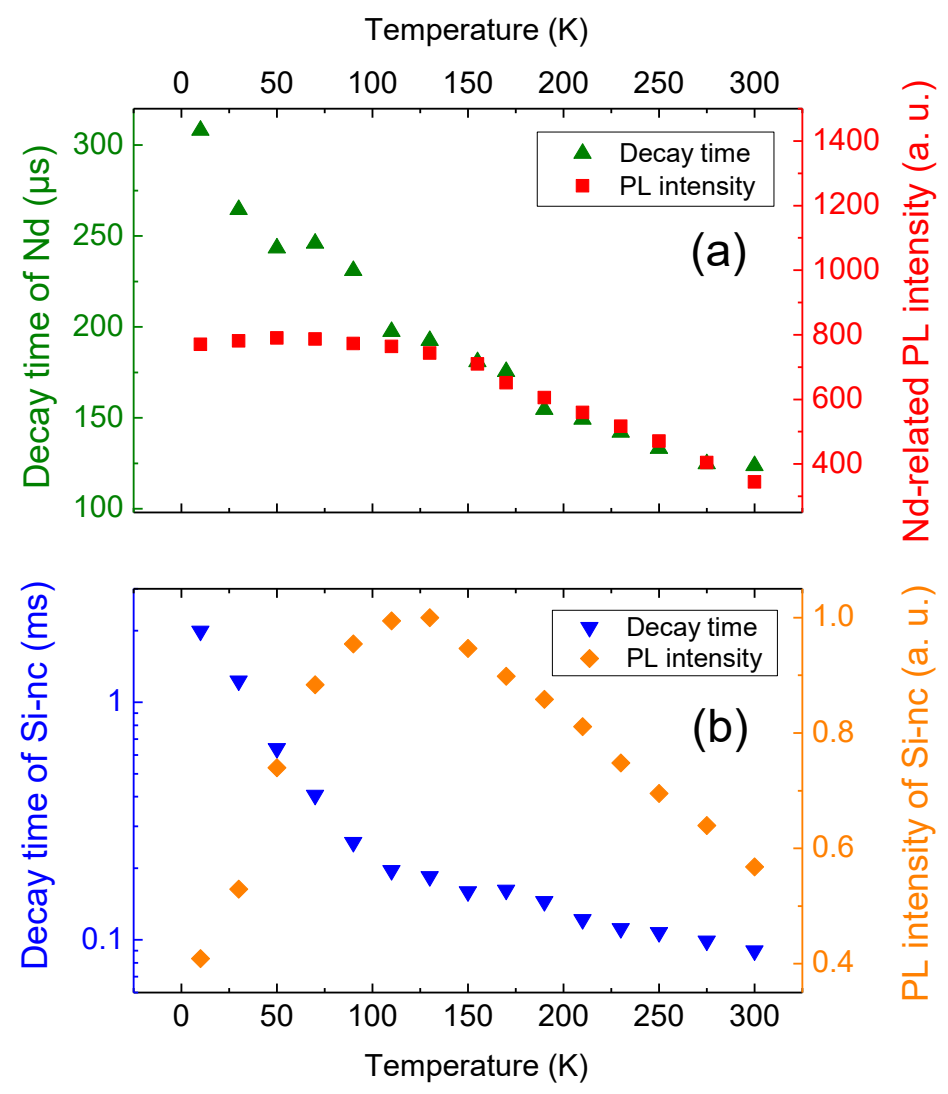

Figure 2: PLintensity and decay time of Nd ions at $920 \mathrm{~nm}$ (a) and Si-nc at $750 \mathrm{~nm}$ (b) as a function of the temperature. The PL intensity is obtained with an indirect excitation at $325 \mathrm{~nm}$. 


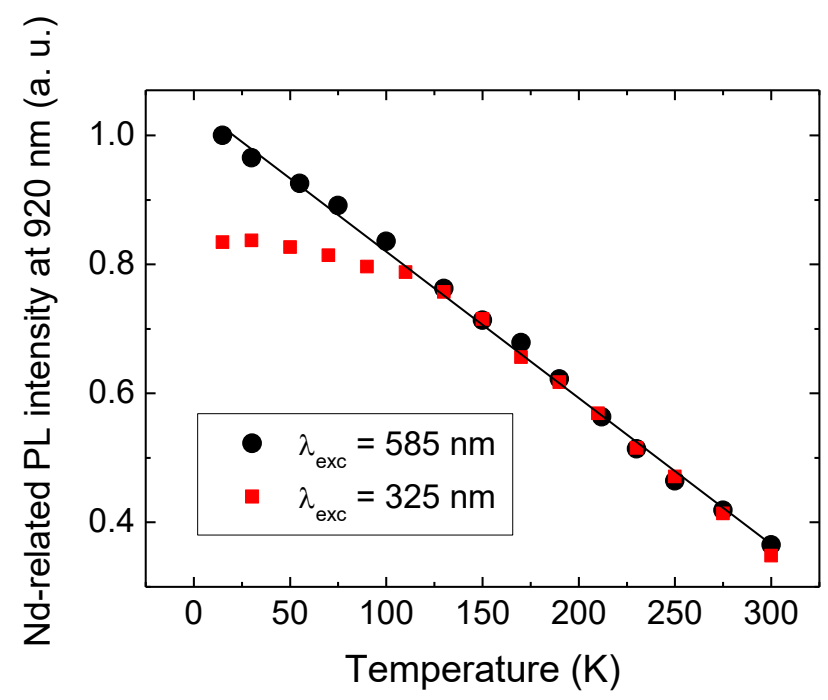

Figure 3: Normalized Nd-related PL intensity as a function of the temperature for direct excitation (at $585 \mathrm{~nm}$ ) and for indirect excitation (at $325 \mathrm{~nm}$ ) of the rare earth ions in a sample containing Sinc. 


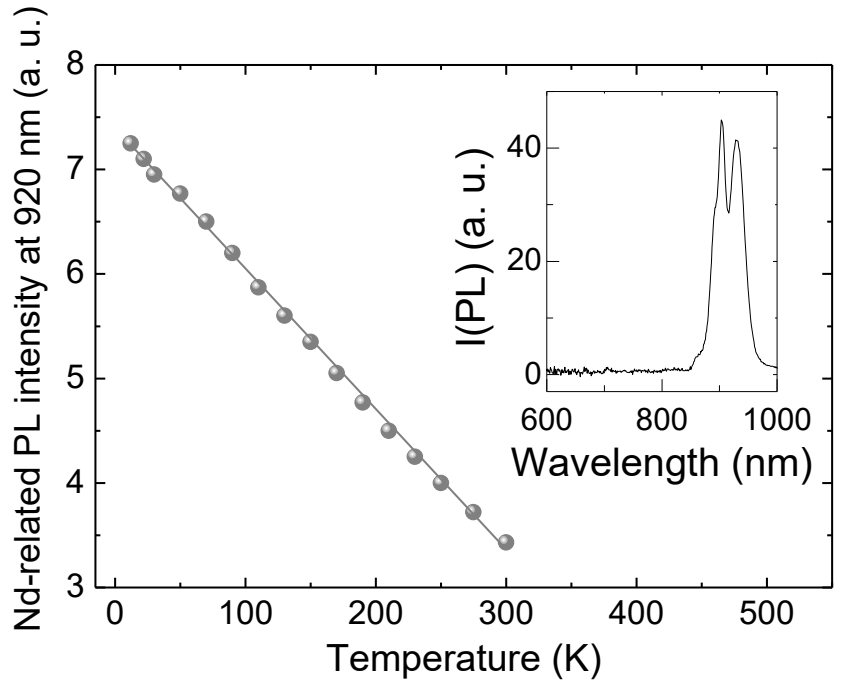

Figure 4: Nd-related PL intensity as a function of the temperature for a sample without Si-nc annealed at $800{ }^{\circ} \mathrm{C}$. The PL intensity is obtained with an indirect excitation at $325 \mathrm{~nm}$. The inset shows the room-temperature PL spectrum of the Nd-doped $\mathrm{SiO}_{1.5}$ sample annealed at $800{ }^{\circ} \mathrm{C}$. 
Figure captions

Figure 1: (Color online) CW-PL spectra of Nd-doped silicon oxide thin films containing $\mathrm{Si}$ nanocrystals as a function of the wavelength for different temperature measurements ranging from $10 \mathrm{~K}$ to $300 \mathrm{~K}$. The spectra are obtained with an indirect excitation at $325 \mathrm{~nm}$.

Figure 2: PL intensity and decay time of $\mathrm{Nd}$ ions at $920 \mathrm{~nm}$ (a) and Si-nc at $750 \mathrm{~nm}$ (b) as a function of the temperature. The PL intensity is obtained with an indirect excitation at $325 \mathrm{~nm}$.

Figure 3: Normalized Nd-related PL intensity as a function of the temperature for direct excitation (at $585 \mathrm{~nm}$ ) and for indirect excitation (at $325 \mathrm{~nm}$ ) of the rare earth ions in a sample containing Sinc.

Figure 4: Nd-related PL intensity as a function of the temperature for a sample without Si-nc annealed at $800{ }^{\circ} \mathrm{C}$. The PL intensity is obtained with an indirect excitation at $325 \mathrm{~nm}$. The inset shows the room-temperature PL spectrum of the Nd-doped $\mathrm{SiO}_{1.5}$ sample annealed at $800{ }^{\circ} \mathrm{C}$. 


\section{Highlights}

- Neodymium-doped silicon oxide thin films have been prepared by evaporation.

- Silicon nanocrystals are generated in the films by annealing post-treatments.

- Under indirect excitation, neodymium ions are sensitized by nanocrystals.

- Below $100 \mathrm{~K}$, the neodymium-related photoluminescence intensity saturates.

- Saturation is explained by a decrease of the coupling efficiency with nanocrystals. 
[1] A. Polman, J. Appl. Phys. 82 (1997) 1-39.

[2] P. G. Kik, M.L. Brongersma, A. Polman, Appl. Phys. Lett. 76 (2000) 2325-2327.

[3] G. Franzò, D. Pacifici, V. Vinciguerra F. Priolo, F. Iacona, Appl. Phys. Lett. 76 (2000) 21672169.

[4] M. Fujii, M. Yoshida, Y. Kansawa, S. Hayaski, K. Yamamoto, Appl. Phys. Lett. 71 (1997) 11981200.

[5] H. Rinnert, G. Wora Adeola, M. Vergnat, Appl. Phys. Lett. 105 (2009) 36101.

[6] S. Y. Seo, M. J. Kim, J. Shin, Appl. Phys. Lett. 83 (2003) 2778.

[7] K. Watanabe, H. Tamaoka, M. Fujii, K. Moriwaki, S.Hayashi, Physica E 13 (2002) 1038.

[8] D. Bréard, F. Gourbilleau, A. Belarouci, C. Dufour, R. Rizk, J. Lumin. 121 (2006) 209.

[9] A. N. MacDonald, A. Hryciw, F. Lenz, A. Meldrum, Appl. Phys. Lett. 89 (2006) 173132.

[10] E. Steveler, H. Rinnert, and M. Vergnat, J. Appl. Phys. 110 (2011) 113518.

[11] A. G. Cullis, L. T. Canham, P. D. J. Calcott, J. Appl. Phys. 82 (1997) 909.

[12] M. L. Brongersma, P. G. Kik, A. Polman, K. S. Min, H. A. Atwater, Appl. Phys. Lett. 76 (2000) 351.

[13] Y. Kanemitsu, T. Ogawa, K. Shiraishi, K. Takeda, Phys. Rev. B 48 (1993) 4883.

[14] V. Vinciguerra, G. Franzò, F. Priolo, F. Iacona, and C. Spinella, J. Appl. Phys. 87 (2000) 8165.

[15] D. Kovalev, H. Heckler, G. Polisski, and F. Koch, Phys. Status Solidi (b) 215 (1999) 871.

[16] H. Rinnert, O. Jambois, and M. Vergnat, J. Appl. Phys. 106 (2009) 023501.

[17] A.M. Hartel, S. Gutsch, D. Hiller, and M. Zacharias, Phys. Rev. B 85 (2012) 165306

[18] H. Rinnert, M. Vergnat, and A. Burneau, J. Appl. Phys. 89 (2001) 237.

[19] E . Steveler, H. Rinnert, X. Devaux, M. Dossot, and M. Vergnat, Appl. Phys. Lett. 97 (2010) 221902.

[20] E. Steveler, H. Rinnert, M. Vergnat, J. Lumin. 150 (2014) 35. 
[21] L. Pavesi and M. Ceschini, Phys. Rev. B 48 (1993) 17625.

[22] C. Delerue, G. Allan, C. Reynaud, O. Guillois, G. Ledoux, and F. Huisken, Phys. Rev. B 73 (2006) 235318.

[23] P. D. J. Calcott, K. J. Nash, L. T. Canham, M. J. Kane, D. Brumhead, J. Phys.: Condens. Matter $5(1993) 91$.

[24] T. Förster, J. S. Kirby-Smith, J. L. Magee, M. Burton, Comparative effects of radiation, Wiley, New-York (1960) 3133. 